\title{
MOLECULAR DYNAMICS STUDY OF TEMPERATURE EFFECTS ON ELECTROKINETIC TRANSPORT IN SI NANOCHANNEL
}

\author{
Bohumir Jelinek* \\ Center for Advanced Vehicular Systems \\ Mississippi State University \\ Mississippi State, Mississippi 39762 \\ bj48@msstate.edu
}

\author{
Sergio D. Felicelli \\ Mechanical Engineering Dept. \\ Mississippi State University \\ Mississippi State, Mississippi 39762 \\ felicelli@me.msstate.edu
}

\author{
Paul F. Mlakar, John F. Peters \\ U.S. Army ERDC \\ 3909 Halls Ferry Rd \\ Vicksburg, Mississippi 39180 \\ Paul.F.Mlakar@erdc.usace.army.mil \\ John.F.Peters@erdc.usace.army.mil
}

\begin{abstract}
We studied temperature effects on the electrokinetic transport in the nanochannel with a slab geometry using a molecular dynamic (MD) model. A simple, previously published system consisting of $\mathrm{Na}^{+}$and $\mathrm{Cl}^{-}$ions dissolved in water and confined between fixed crystalline silicon walls with negatively charged inner surfaces in an external electric field was chosen as a benchmark. Dependence of the fluid flow on the temperature and thermostating techniques was examined. The variation of resulting areal mass flux density and molecular velocity profiles across the channel with initial conditions was explored to asses the statistical significance of the results. It was found that, at least at the size scale, surface charge density, electric field and ionic concentrations of the benchmark system, the water flux and even its direction are directly controlled by temperature.
\end{abstract}

\section{INTRODUCTION}

Nanoscale numerical models of electro-osmosis [1-9] provide insight into the interplay of different transport mechanisms, help improve current technological devices, and guide the design of new technology based on the principles of electrokinetic transport.

We present a parametric study of the fluid flow and ion species transport under an electric field in nanochannels typically found in heterogeneous porous media. Although the continuum conservation equations can be applied to microscale chan-

*Address all correspondence to this author.
Table 1. Parameters of Lennard-Jones potentials, $\sigma_{i j}$ in the units of $\AA$, $\epsilon_{i j}$ in $\mathrm{cal} / \mathrm{mol}$.

i-j O-O O-Si O-Na O-Cl Si-Si Si-Na Si-Cl Na-Na Na-Cl Cl-Cl

\begin{tabular}{lllllllllll}
\hline$\sigma_{i j}$ & 3.17 & 3.27 & 2.86 & 3.75 & 3.39 & 2.95 & 3.88 & 2.58 & 3.38 & 4.45
\end{tabular}

$\begin{array}{lllllllllll}\epsilon_{i j} & 155 & 301 & 47.9 & 129 & 584 & 92.9 & 249 & 14.8 & 39.6 & 106\end{array}$

nels, the main driving force of electrokinetic transport occurs in an electric double layer (EDL) at the solid liquid interface with dimensions that can be comparable to intermolecular distances. Therefore, molecular dynamics (MD) [10-13] simulations were applied to analyze the interaction between ions, water molecules and wall atoms in the EDL region. Classical Lennard-Jones (LJ) force fields and Coulomb electrostatic interactions with Simple Point Charge Extended (SPC/E) model of water were used to represent the interactions between ions, water molecules and channel wall atoms. Time averaged mass flux density, velocity, and concentration profiles of water molecules and ionic species were obtained from MD simulation.

\section{MD potentials}

Combination of Lennard-Jones (LJ) and Coulomb electrostatic potentials was used to describe potential energy of the atomic configuration. $\mathrm{LJ}$ contribution of atoms $i$ and $j$ to the 


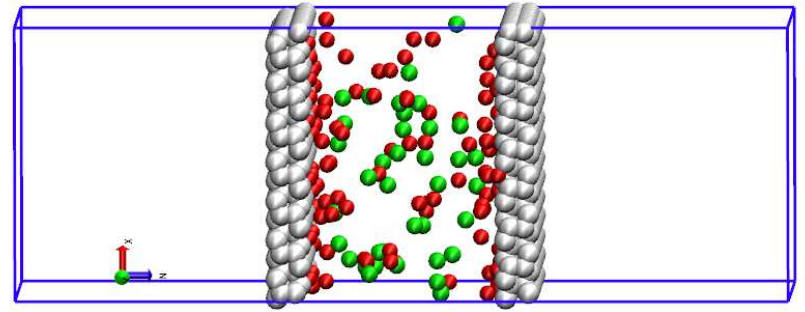

Figure 1. Simulation box, without water molecules. Si wall atoms are gray, $\mathrm{Na}^{+}$ions red and $\mathrm{Cl}^{-}$ions green. Electric field is applied in the positive $\mathrm{x}$ (upward) direction.

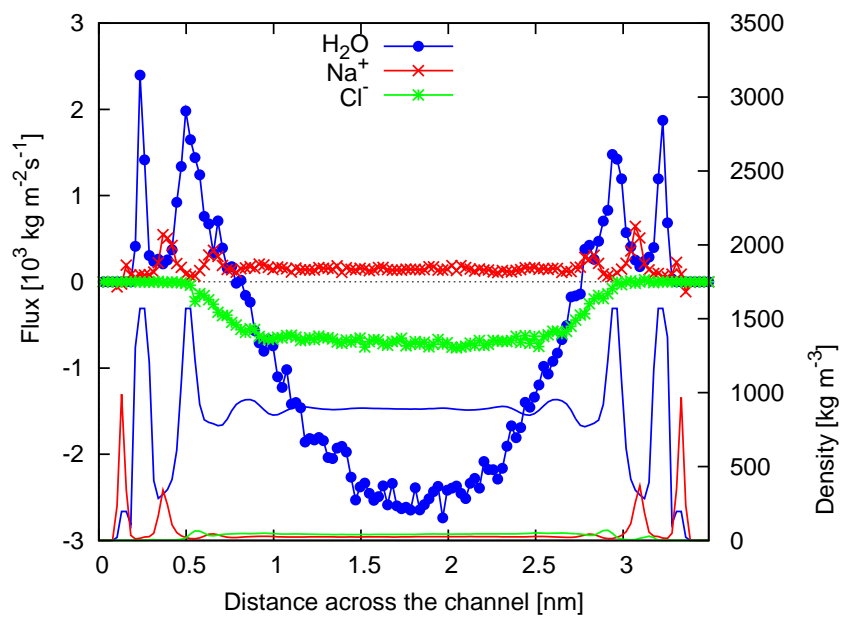

Figure 2. Time averaged $x$-component of areal mass flux and density profile across the channel width from $22 \mathrm{~ns}$ simulation at $\mathrm{T}=300 \mathrm{~K}$. Range of distance axis starts at left and ends at right innermost channel wall, as shown in Figure 1. Negative flux means flux in the direction opposite to the applied electric field.

total potential energy is specified in Eq. (1).

$$
V_{L J}(r)=4 \epsilon_{i j}\left[\left(\frac{\sigma_{i j}}{r}\right)^{12}-\left(\frac{\sigma_{i j}}{r}\right)^{6}\right]
$$

The parameters $\epsilon_{i j}$ and $\sigma_{i j}$ depend on atomic species of $i$ th and $j$ th atom. Coulomb electrostatic potential energy contribution is calculated from Eq. (2).

$$
V_{C}(r)=\frac{1}{4 \pi \varepsilon_{0}} \frac{q_{i} q_{j}}{r}
$$

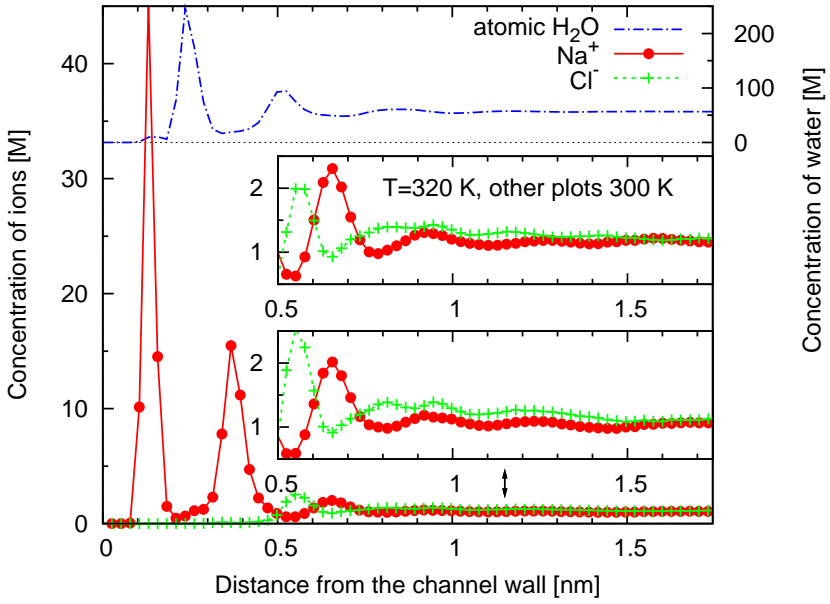

Figure 3. Average concentrations of water and ions across left half of the channel. The plot in the center shows concentrations at $\mathrm{T}=320 \mathrm{~K}$, remaining plots are at $\mathrm{T}=300 \mathrm{~K}$.

The $\varepsilon_{0}$ is vacuum permittivity, $q_{i}$ and $q_{j}$ are electric charges of $i$ th and $j$ th atom and $r$ is the distance between $i$ th and $j$ th atom.

We used potential parameters from the GROMACS [14] force field listed in the Table 1.

\section{Simulation setup}

We reproduced the system of Qiao and Aluru [7], summary of which will be provided now. Dimensions of the solution region were $4.66 \times 4.22 \times 3.49 \mathrm{~nm}$. Channel walls, perpendicular to the $\mathrm{z}$ axis, were formed by four [111] oriented layers of $\mathrm{Si}$ atoms in a diamond crystal structure, each wall $3.92 \mathrm{~nm}$ thick. Periodic boundary conditions were applied in the $\mathrm{x}, \mathrm{y}$ and $\mathrm{z}$ directions, while the size of simulation cell in the $\mathrm{z}$ direction was extended to three times outermost-to-outermost wall layer distance (4.37 $\mathrm{nm})$ to mitigate electrostatic interactions of periodic images in the $\mathrm{z}$ direction. The PME (Particle-Mesh-Ewald) method [15] with a slab correction [16] in the $\mathrm{z}$ direction was used for long range electrostatics. Electric field was applied in the positive $x$ direction.

To generate initial atomic positions, charged walls were generated (924 uncharged $\mathrm{Si}$ atoms and 308 negatively charged), 2290 water molecules were inserted avoiding close contacts (memory intensive solvation process), and randomly selected 146 water molecules were replaced by $108 \mathrm{Na}^{+}$and $38 \mathrm{Cl}^{-}$ions. To make the system electrically neutral, 70 electrons charge was distributed discretely on each innermost wall Si surface atom, resulting in $-0.227273 \mathrm{C}$ per atom charge, or surface charge density of $-0.285 \mathrm{C} / \mathrm{m}^{2}$.

First, the energy minimization of the system was performed using conjugate gradient method. Then, the system was equi- 

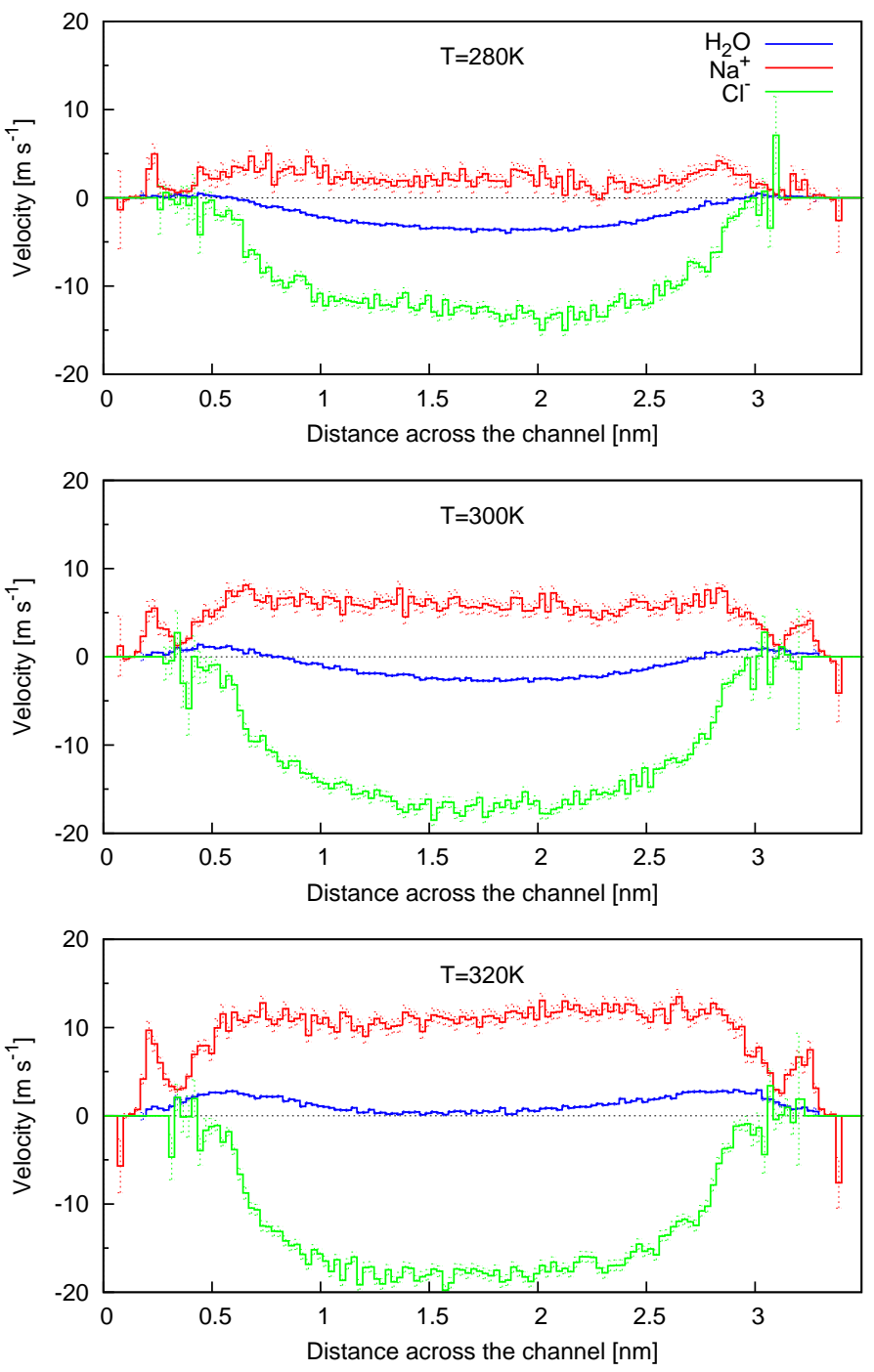

Figure 4. Velocity profiles at $\mathrm{T}=280 \mathrm{~K}$ (top), $300 \mathrm{~K}$ and $320 \mathrm{~K}$ (bottom). Note that the temperature increase changes the water flow direction along the channel.

librated by 2 ns MD simulation without electric field. The timestep of $2 \mathrm{fs}$ was used for the leap-frog integration of Newton's equations of motion. Resulting configuration, excluding water molecules, is shown in Figure 1. Finally, a 22 ns MD run was performed with external electric field of $0.55 \mathrm{~V} / \mathrm{nm}$. The SETTLE [17] algorithm was used to constrain bonds of water molecules. Positions of Si wall atoms were fixed and the solution temperature was controlled by the Nosé-Hoover $[18,19]$ thermostat. The Berendsen [20] and velocity-rescaling [21] thermostats were also tested, but changes of the velocity and flux profiles were insignificant.
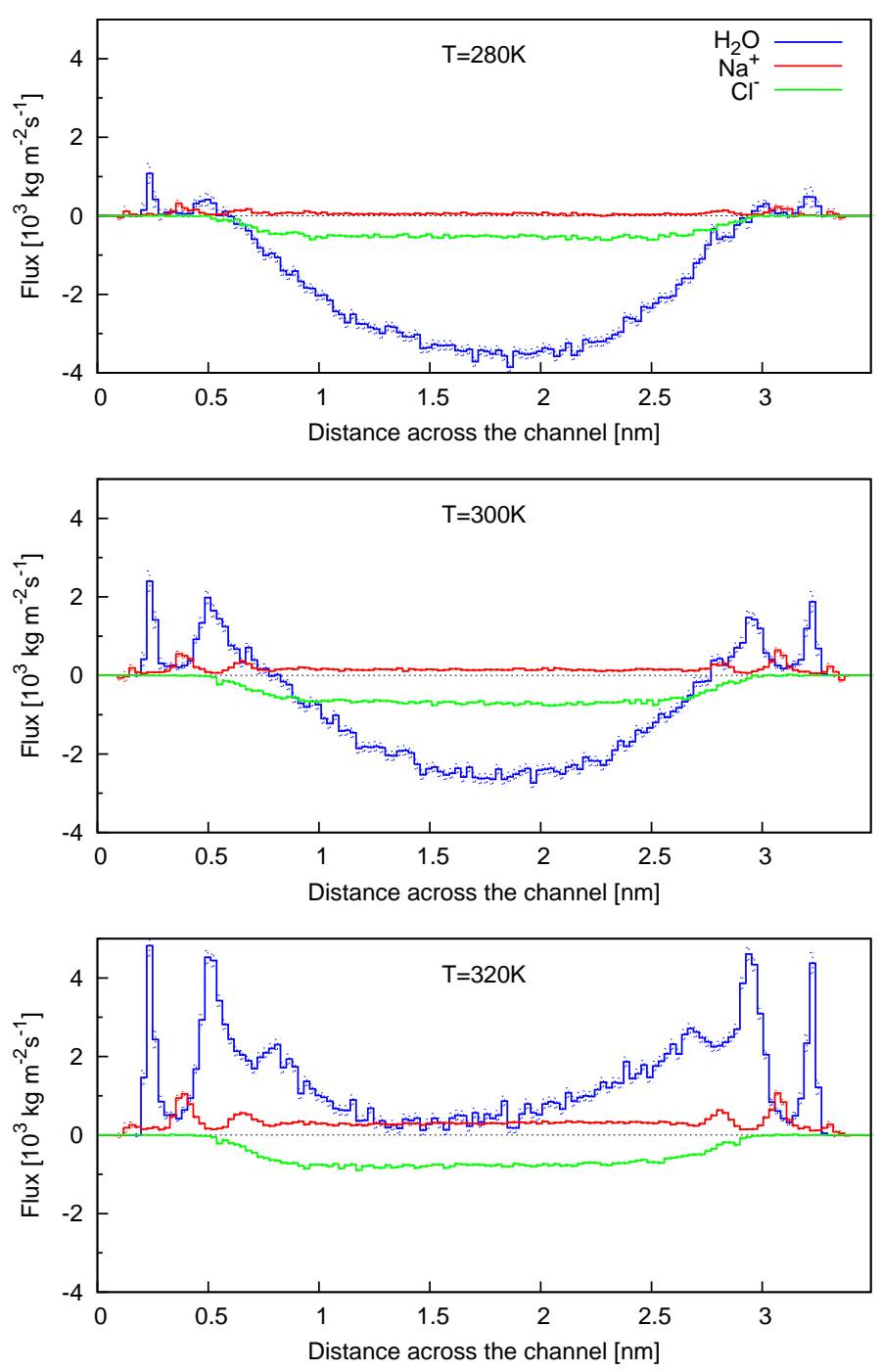

Figure 5. Mass flux profiles at $\mathrm{T}=280 \mathrm{~K}$ (top), $300 \mathrm{~K}$ and $320 \mathrm{~K}$ (bottom). Note that the temperature increase changes the water flow direction along the channel.

\section{Concentrations and flow profiles}

Atomic concentration profile on Figure 3, which agrees very well with the one of Qiao and Aluru [7], manifests formation of alternatively charged layers of atoms parallel to the channel walls. Negatively charged Si wall attracts positively charged $\mathrm{Na}^{+}$ions and slightly positively charged $(0.4238 \mathrm{C}$ /atom for SPC/E water) $\mathrm{H}$ atoms from $\mathrm{H}_{2} \mathrm{O}$ molecules, forming the first near-wall concentration peak. Since this positively charged layer screens off the negatively charged wall for atoms further inside the channel, the adjacent layer of negative $\mathrm{Cl}^{-}$ions and slightly positively charged $\mathrm{O}$ atoms $(-0.8476 \mathrm{C}$ for SPC/E water) from water molecules is formed. Five noticeable layers with alter- 
nating charge sign are formed until concentrations become more balanced (around $1 \mathrm{M}$ concentration) towards the channel center.

The flux and mass density profile on Figure 2 show that main contributor to the mass flux are regions with high density of water molecules. Flux of water was calculated by replacing movement of atoms in water molecule by its center of mass (COM) movement. Since the net charge of water molecule is zero, its COM will not move in the presence of external electric field. Water molecules can only be dragged by ions or by hydrogen bonding to neighboring water molecules.

Two peaks in water flux result from two water layers being dragged by two $\mathrm{Na}^{+}$layers near the channel wall. The competing water moving mechanism in the channel center, causing the flow reversal at lower temperatures, is water being dragged by $\mathrm{Cl}^{-}$ions. $\mathrm{Cl}^{-}$ions move faster than $\mathrm{Na}^{+}$ions (Figure 4 displays velocities), since their $\mathrm{LJ}$ potential parameters are constructed to reproduce higher experimental mobility of $\mathrm{Cl}^{-}$ions. Interestingly, the water movement by near-wall $\mathrm{Na}^{+}$layers dominates at higher temperatures, as presented in the next section.

\section{Temperature effects}

A significant temperature control of the flow was observed. Figures 4 and 5 show the dependence of the flow profiles of ions and water on the temperature. It appears that the fluid flux and even its direction are directly controlled by the temperature. At lower temperatures, flow reversal of water is observed, while at higher temperatures, the water flows in the direction expected by a standard EDL model. Our hypothesis, explaining the temperature dependence of the flux, is that the stagnant layer of $\mathrm{Na}^{+}$ ions adsorbed at the channel wall is freed at higher temperatures (by thermal motion) and moves in the direction of electric field, speeding up the movement of the near-wall layers of water that are dragged by $\mathrm{Na}^{+}$layer. The near-wall water layers then drag the adjacent layers in the channel center by hydrogen bonding, outperforming the competing mechanism of water dragged in the negative $\mathrm{x}$ direction by faster $\mathrm{Cl}^{+}$ions in the channel center.

In order not to alter the field direction velocity component by thermostating (thermostat adjusts the velocity to maintain the temperature and its proper fluctuations), we compared our GROMACS simulation to LAMMPS [22] results with profileunbiased thermostat [23] that preserves the X-component velocity profile across the channel. Velocity profiles were almost identical to those obtained from GROMACS by thermostating all velocity components, even though LAMMPS is using PPME [24] method for long range electrostatics.

Moving averages of the COM velocities of water and ions are shown in Figure 6. Even though the sample intervals for moving averages are quite large, significant fluctuations in COM velocities are observed. The duration of simulation and the number of simulations performed to obtain the time averages are important since the small size of the system causes the thermal fluctua-

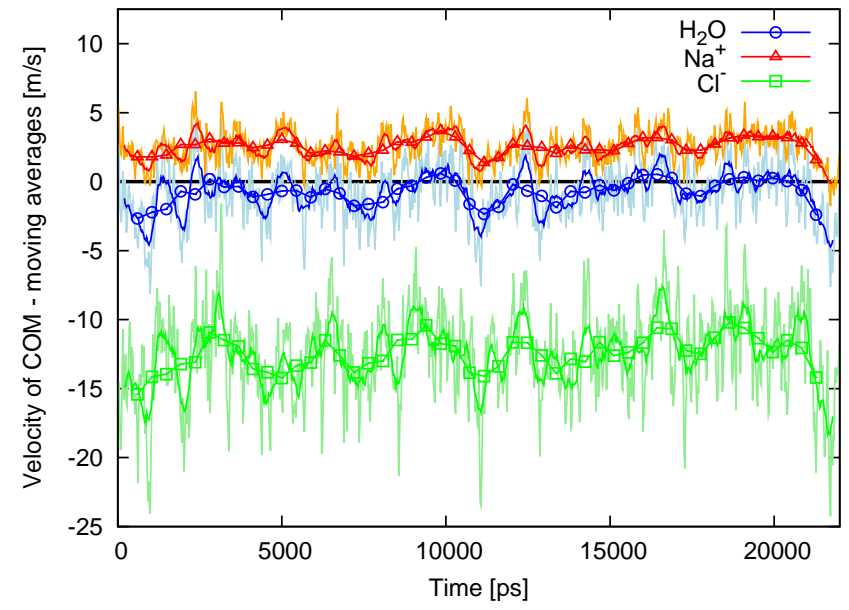

Figure 6. Moving averages of COM velocities of all water molecules, $\mathrm{Na}^{+}$ions, and $\mathrm{Cl}^{-}$ions.

tions in velocities to be an important factor, considered hereafter.

\section{Statistical significance}

We explored the effects of initial conditions by changing the initial velocity seeds for ten simulations with each single and double precision compiled GROMACS. The average COM velocity of water (moving averages from sample simulation are shown in Figure 6) and sample standard deviation from ten randomly initialized $22 \mathrm{~ns}$ single precision GROMACS calculations at $\mathrm{T}=300 \mathrm{~K}$ were $-5.6+/-2.5 \mathrm{~ms}^{-1}$, while the results from double precision calculations were $-3.8+/-3.3 \mathrm{~ms}^{-1}$. We concluded that, for the quantities we are interested in, the simulations using the single precision GROMACS are satisfactory. The standard deviations of samples from both single and double precision models are acceptable to demonstrate temperature effects.

\section{Conclusions}

Our results show a flux of highly concentrated $\mathrm{Na}^{+}$ions and water in the layer near the channel wall, that corresponds to the common electric double layer justification of the electroosmotic phenomena. It was found that at the size scale, surface charge density, electric field and ionic concentrations of the investigated system, the fluid flux and its direction are directly controlled by the temperature.

More detailed studies of electric field strength effects, evaluation of the influence of surface charge density, species concentrations, channel width and wall material are in progress. Longterm goal of this work is to supplement the continuum modeling of the electrokinetic transport in concrete nanopores by the information from classical MD simulations. 


\section{ACKNOWLEDGMENT}

This work was funded by the US Army Corps of Engineers through contract number W912HZ-09-C-0024. Computational resources at the MSU $\mathrm{HPC}^{2}$ center were utilized. Computational packages GROMACS [14, 25, 26] versions 4.0.4 and LAMMPS [22] were used to perform MD simulations. Figure 1 was made using Visual Molecular Dynamics [27] package.

Permission to publish this material was granted by the Director of the Geotechnical and Structures Laboratory of the Engineer Research and Development Center, research and development organization for the U.S. Army Corps of Engineers.

\section{REFERENCES}

[1] Liu, Y., Oh, K., Bai, J. G., Chang, C.-L., Yeo, W., Chung, J.-H., Lee, K.-H., and Liu, W. K., 2008. "Manipulation of nanoparticles and biomolecules by electric field and surface tension". Computer Methods in Applied Mechanics and Engineering, 197(25), Apr., pp. 2156-2172.

[2] Patankar, N. A., and Hu, H. H., 1998. "Numerical simulation of electroosmotic flow". Analytical Chemistry, 70(9), pp. 1870-1881.

[3] Qiao, R., and Aluru, N. R., 2004. "Multiscale simulation of electroosmotic transport using embedding techniques". Int. J. Multisc. Comp. Eng., 2(2), pp. 173-188.

[4] Rotenberg, B., Marry, V., Dufreche, J.-F., Giffaut, E., and Turq, P., 2007. "A multiscale approach to ion diffusion in clays: Building a two-state diffusion-reaction scheme from microscopic dynamics". Journal of Colloid and Interface Science, 309(2), May, pp. 289-295.

[5] Joseph, S., and Aluru, N., 2006. "Hierarchical multiscale simulation of electrokinetic transport in silica nanochannels at the point of zero charge". Langmuir, 22(21), pp. 90419051.

[6] $\mathrm{Hu}, \mathrm{G}$., and Li, D., 2007. "Multiscale phenomena in microfluidics and nanofluidics". Chem. Eng. Science, 62(13), July, pp. 3443-3454.

[7] Qiao, R., and Aluru, N. R., 2004. "Charge inversion and flow reversal in a nanochannel electro-osmotic flow". Phys. Rev. Lett., 92(19), May, p. 198301.

[8] Huang, D. M., Cottin-Bizonne, C., Ybert, C., and Bocquet, L., 2007. "Ion-specific anomalous electrokinetic effects in hydrophobic nanochannels". Phys. Rev. Lett., 98(17), p. 177801.

[9] Lorenz, C., Crozier, P., Anderson, J., and Travesset, A., 2008. "Molecular Dynamics of Ionic Transport and Electrokinetic Effects in Realistic Silica Channels". J. Phys. Chemistry C, 112(27), pp. 10222-10232.

[10] Rapaport, D., 2004. The Art of Molecular Dynamics Simulation. Cambridge University Press.

[11] Frenkel, D., and Smit, B., 2002. Understanding Molecular
Simulation: From Algorithms to Applications. Academic Press.

[12] Allen, M., and Tildesley, D., 1990. Computer simulation of liquids. Oxford University Press, USA.

[13] Feynman, R., Leighton, R., and Sands, M., 1963. The Feynman Lectures on Physics; Vol. I. Addison-Wesley.

[14] Hess, B., Kutzner, C., van der Spoel, D., and Lindahl, E., 2008. "Gromacs 4: Algorithms for highly efficient, loadbalanced, and scalable molecular simulation". Journal of Chemical Theory and Computation, 4(3), pp. 435-447.

[15] Essmann, U., Perera, L., Berkowitz, M., Darden, T., Lee, H., and Pedersen, L., 1995. "A smooth particle mesh Ewald method". J. Chem. Physics, 103(19), pp. 8577-8593.

[16] Yeh, I., and Berkowitz, M., 1999. "Ewald summation for systems with slab geometry". J. Chem. Physics, 111, p. 3155 .

[17] Miyamoto, S., and Kollman, P., 1992. "SETTLE: an analytical version of the SHAKE and RATTLE algorithm for rigid water models". J. Comp. Chemistry, 13(8).

[18] Nosé, S., 1984. "A unified formulation of the constant temperature molecular dynamics methods". J. Chem. Physics, 81(1), pp. 511-519.

[19] Hoover, W. G., 1985. "Canonical dynamics: Equilibrium phase-space distributions". Phys. Rev. A, 31(3), Mar., pp. 1695-1697.

[20] Berendsen, H. J. C., Postma, J. P. M., van Gunsteren, W. F., DiNola, A., and Haak, J. R., 1984. "Molecular dynamics with coupling to an external bath". J. Chem. Physics, 81(8), pp. 3684-3690.

[21] Bussi, G., Donadio, D., and Parrinello, M., 2007. "Canonical sampling through velocity rescaling". J. Chem. Physics, 126(1), p. 014101.

[22] Plimpton, S. J., 1995. "Fast Parallel Algorithms for ShortRange Molecular Dynamics". J. Comp. Physics, 117(1), pp. 1-19.

[23] Evans, D. J., and Morriss, G. P., 1986. "Shear thickening and turbulence in simple fluids". Phys. Rev. Lett., 56(20), May, pp. 2172-2175.

[24] Plimpton, S. J., Pollock, R., and Stevens, M., 1997. "Particle-mesh ewald and rrespa for parallel molecular dynamics simulations". In Procs. of the 8th SIAM Conference on Parallel Processing for Scientific Computing, pp. 8-21.

[25] Lindahl, E., Hess, B., and van der Spoel, D., 2001. "GROMACS 3.0: a package for molecular simulation and trajectory analysis". J. Mol. Mod., 7(8), pp. 306-317.

[26] Berendsen, H., Van der Spoel, D., and Van Drunen, R., 1995. "GROMACS: a message-passing parallel molecular dynamics implementation". Computer Physics Communications, 91(1-3), pp. 43-56.

[27] Humphrey, W., Dalke, A., and Schulten, K., 1996. "VMD - Visual Molecular Dynamics". J. Mol. Graphics, 14, pp. 33-38. 